\title{
Politique
}

Politique

\section{Christiane Olivier, Les enfants de Jocaste; l'empreinte de la mère. Paris, Denoël — Gonthier, 1980}

\section{Nathaly Gagnon}

Numéro 5, hiver 1984

Femmes et pouvoir

URI : https://id.erudit.org/iderudit/040456ar

DOI : https://doi.org/10.7202/040456ar

Aller au sommaire du numéro

\section{Éditeur(s)}

Société québécoise de science politique

ISSN

0711-608X (imprimé)

1918-6584 (numérique)

Découvrir la revue

Citer ce compte rendu

Gagnon, N. (1984). Compte rendu de [Christiane Olivier, Les enfants de Jocaste; l'empreinte de la mère. Paris, Denoël - Gonthier, 1980]. Politique, (5), 157-162. https://doi.org/10.7202/040456ar d'utilisation que vous pouvez consulter en ligne.

https://apropos.erudit.org/fr/usagers/politique-dutilisation/ 
Christiane Olivier, Les enfants de Jocaste; l'empreinte de la mère. Paris, Denoël - Gonthier, 1980.

«Il y a le discours analytique: recherché, compliqué, étudié pour vous écarter, vous semer, vous éberluer vous qui n'êtes pas analystes...

Il y a le discours féministe: discours coloré, imagé, sexué, fait pour que vous entriez, compreniez, même si vous n'êtes pas féministe, d'autant que vous n'êtes pas féministe...

Et il y a ceux qui ne se reconnaissent ni dans l'un ni dans l'autre parce que, de toute façon, ils refusent d'être extrémistes.

Me tenir entre ces deux discours, ne pas m'isoler en adoptant le premier, ne pas vous submerger en parlant le second. Parler le langage du milieu, celui qui ne met de côté ni l'affect, ni l'intellect. Être femme et analyste, c'està-dire porter les deux extrêmes, garder ensemble l'émoi et le verbe, refuser d'être ou plus femme ou plus analyste, refuser de me diviser ou de me spécialiser." (p. 5)

Tel est le projet de Christiane Olivier, femme psychanalyste qui tente d'étudier ce personnage absent de la théorie freudienne: Jocaste, mère d'Oedipe parce que, dit-elle «il est nécessaire de repenser la théorie de l'inconscient, avec l'aide des femmes, grâce à leur parole.» (p. 12) 
Jusqu'à très récemment, la psychanalyse ne s'était pas préoccupée du désir de la femme puisque les prémisses de cette théorie étaient uniquement masculines. Freud lui-même, s'utilisant comme sobjet de recherches n'a pu définir la femme qu’à partir de son environnement social, soit celui de la petite-bourgeoisie du XIX ${ }^{e}$ siècle dont les rôles étaient bien établis. Donc, constatant l'infériorité sociale des femmes, Freud l'a consacrée de façon scientifique:

«Il (Freud) l'a vue se taire en présence de l'homme et en a conclu à son incapacité de sublimation intellectuelle. Il l'a vue servir l'homme et l'a imaginée masochiste. Il l'a vue s'occuper de l'enfant et aussitôt l'a assignée à maternité pour combler son manque (selon la fameuse équation: pénis=enfant). (p. 15)

Le droit à la jouissance sans passer par la maternité a permis aux femmes de rendre à la fonction sexuelle la place qu'elle doit avoir. C'est donc une dénonciation de l'antiféminisme de Freud qui permettra à Christiane Olivier de corriger l'erreur fondamentale au sujet de la sexualité des femmes; en utilisant donc l'origine des théories freudiennes tout en en rejetant les conclusions.

La première erreur de Freud consiste à établir une ‘symétrie` dans l'évolution des petits garçons et des petits filles tout en établissant à priori la suprématie masculine. C'était nier au départ la «disymétrie fondamentale « qui existe entre les sexes et obliger les enfants-filles à accepter la supériorité mâle. Freud en arrive donc à des affirmations invraisemblables concernant la sexualité féminine dans ses Trois essais sur la sexualité (1905) telles que: l'envie du pénis et le renoncement au clitoris. Mais d'envie du vagin, envie de vulve, envie de sein... on n'en voit pas. Les termes mêmes utilisés par Freud (envie, jalousie, renoncement, absence de... etc.) sont caractéristiques d'une vision qui ne voit la femme que comme l'envers de l'homme. Faisant un saut dans la littérature et en art, Christiane Olivier nous transporte dans un monde d'hommes qui n'a cessé de faire l'éloge des attributs féminins que l'homme ne possède pas: gorge, seins, taille fine (elle cite poètes 
et écrivains tels que Molière, Ronsard, Eluard, Beaudelaire, Miller, ainsi que des artistes: Picasso, Chagall, Dali, etc.) Et elle fait remarquer à juste titre que côté littérature et art féminins c'est le silence absolu sur cet objet que nous convoitons tant: le pénis.

"Nous avions un sexe chargé de tant d'événements, d'aventures, et d'expériences que l'homme aurait pu en pâlir d'envie et voilà que c'est nous, si riches, dont on a réussi à faire des envieuses." (Annie Leclerc, Parole de femme, Ed. Grasset, Paris, p. 51 , cité en p. 28).

Quant au renoncement au clitoris, il ne résiste pas à l'analyse. C'est comme si nous n'existions pas en tant qu'entité et avions besoin d'une partie de l'anatomie masculine pour être totales. Les femmes adultes qui jouissent encore avec leur clitoris (et non avec leur vagin) sont donc prédisposées, selon Freud, aux névroses et à l'hystérie. La jouissance est réduite à celle de l'autre: l'homme rend la femme prisonnière de son pénis, "valeur première du système phallocratique» (p. 33). On sait maintenant, grâce aux observations scientifiques et à la parole des femmes (Rapport Hite) que le clitoris est «le premier élément inaugurant toute autre jouissance». (p. 34)

Pourtant, Christiane Olivier mentionne que les dernières interrogations de Freud concernant la sexualité féminine auraient pu ouvrir la voie à des conclusions moins sexistes et qu'il faut en vouloir à ses successeurs, hommes ou femmes qui ont préféré s'en tenir à ses premières théories et ont contribué à prôner des inepties du «trop petit pénis et de l'infériorité féminine». (p. 47)

Quant au «complexe d'Oedipe`, un problème se pose: l'éveil de la sexualité de l'enfant se fait par la mère; pour le garçon, le premier objet de désir est (incestueux〉, mais pour la fille, cette dernière n'a pas de premier objet d'amour car «rares sont les pères qui vivent à la maison pouponnant leur fille.» (p. 59) Puisque nous n'en sommes pas encore arrivés à l'ère où les pères ne refuseront plus de paterner leur enfant, nous ne pouvons qu'examiner «la constitution des caractères de l'homme et de la femme, mais tous 
deux face à la mère dans la famille nucléaire et partriarcale actuelle.» (p. 59)

Le petit garçon, dès la naissance, a pour objet d'amour sa mère ou la femme qui l'élève. Cette femme, quant à elle, porte fièrement ce fils qui la complète. Pourtant, cette symbiose ne durera pas car, selon Christiane Olivier «la femme a inconsciemment du mal à renoncer au seul mâle qu'elle ait jamais eu avec elle: son père lui ayant fait défaut et son mari étant le plus souvent absent. Le petit garçon doit surmonter là une difficulté supplémentaire (non décrite par Freud) car il doit s'échapper de l'oedipe contre sa mère qui ne veut ni qu'il s'éloigne ni qu'il la quitte. C'est là que commence la plus longue et la plus subtile des guerres contre le désir féminin, c'est là que le garçon entre dans la guerre oedipienne des sexes avec sa mère.» (p. 61) Cette évolution de la relation mère-garçon laissera une tache indélébile chez le garçon qui refusera, par la suite, toute autre symbiose avec une autre femme. Il se méfiera à jamais des femmes, deviendra misogyne tout cela parce qu'il a dû se défaire de la femme qu'il a le plus aimée et dont il a été le plus aimé. Tout cela parce que la femme joue seule le rôle d'éducatrice dans la famille et que cette lutte mère-fils se perpétue: «une femme creuse pour une autre le sillon de la misogynie.» (p. 64)

La petite fille, de son côté, n'est pas un objet sexuel satisfaisant pour la mère: on l'aime comme une enfant, on ne la désire pas comme corps de fille.

"Ainsi la petite fille est niée dans sa propre sexualité d'enfant et renvoyée à sa sexualité future de femme; elle a à taire ce qu'elle EST: une petite fille clitoridienne, et elle doit croire à ce qu'elle N'EST PAS: une femme vaginale». (p. 66)

On la verra donc jouer à la femme, et ce, toute sa vie: il lui faut toujours rajouter quelque chose, elle n'est jamais satisfaite de ce qu'elle est: on lui demande de développer son identification plutôt que son identité. Et sa jalousie ne prend pas naissance dans 
l'envie du pénis «mais plutôt dans la comparaison de son corps avec celui de la mère».

Ce désir de plaire à l'homme, l'importance accordée au regard de l'homme d'une part, rendront difficile l'accession aux valeurs féministes qui n'accordent pas de valeur au jugement de l'homme, et d'autre part, attiseront la rivalité entre femmes, rivalité qui a pris naissance avec leur mère.

«Et les femmes ont beaucoup de mal à traverser leur méfiance les unes vis-à-vis des autres, la «sororité» n'est pas une évidence et requiert de renoncer à l'existence reçue de l'extérieur pour adopter celle venue de l'intérieur, mouvement très inhabituel pour une femme." (p. 70)

Par exemple, pour Christiane Olivier, une anorexique est une femme ‘pour elle` qui refuse d'être ‘pour les autres`, qui veut échapper au Désir. Comme si la femme devait choisir entre le Corps et l'Esprit.

«Il semble que ce soit en renonçant délibérément à 'plaire) que les femmes en ce moment se mettent à parler, écrire, dessiner, chanter... Curieux tout de même! Il y aurait donc bien un rapport de forces inversé entre le splaire) et le 'savoir , entre la femme-objet et la femme-intellectuelle? L'idéal social se trouvant chez celle qui tient l'équilibre périlleux entre ces deux femmes». (p. 71)

Mais nous souffrons de ce que nous avons contribué à engendrer et en continuant à revendiquer la garde de l'enfant, les femmes perpétuent le système phallocrate. Les femmes doivent renoncer et les hommes prendre leur place du ‘pouvoir oedipien`.

Christiane Olivier poursuit sa démonstration à l'aide de nombreux exemples de la naissance jusqu'à l'âge adulte, décrivant ainsi le processus qui mène aux postulats mentionnés précédemment. Son expérience de psychanalyste, les exemples puisés à la source, renforcent son énoncé. Elle cite également les recherches de femmes sur différents aspects du sexisme (Marina Yaguello, Hélène Cixous, Simone de Beauvoir, Luce Irigaray, Kate Millett, Benoîte Groulx, etc.).

Ce livre, loin de culpabiliser les femmes (comme l'ont suggéré certaines critiques depuis sa parution), les mères en particulier, 
propose plutôt l'avènement d'une famille et d'une éducation où l'enfant trouverait dès sa naissance deux référents: un masculin, un féminin, supports à l'identification et à l'identité et ce, afin d'assurer une «égalité des rôles dans la différence des sexes». L'enfant pourrait alors concevoir que la différence sexuelle n'engendre pas une différence des pouvoirs "concept qui sert de base à la guerre actuelle entre hommes et femmes». (p. 192) Un livre clair qui démystifie la psychanalyse freudienne à l'égard des femmes et le rôle de maternage qui contribue à perpétuer le clivage entre les sexes. À mon avis, un livre essentiel pour enfin saisir l'ampleur de l'inconscient sur nos comportements (d'hommes et de femmes) vue par une femme qui a osé secouer la tradition psychanalytique.

Nathaly Gagnon

Université du Québec à Montréal 